\title{
Genetic underpinnings of affective temperaments: a pilot GWAS investigation identifies a new genome-wide significant SNP for anxious temperament in $A D G R B 3$ gene
}

\author{
Xenia Gonda (10 1,2, Nora Eszlari 1,3, Dora Torok ${ }^{3}$, Zsofia Gal ${ }^{3}$, Janos Bokor ${ }^{4}$, Andras Millinghoffer ${ }^{5,6}$, Daniel Baksa ${ }^{3,7}$, \\ Peter Petschner (1,3,8 , Peter Antal ${ }^{5}$, Gerome Breen $\mathbb{1}^{9}$, Gabriella Juhasz $\mathbb{0}^{1,3,7}$ and Gyorgy Bagdy ${ }^{1,3}$
}

\begin{abstract}
Although recently a large-sample GWASs identified significant loci in the background of depression, the heterogeneity of the depressive phenotype and the lack of accurate phenotyping hinders applicability of findings. We carried out a pilot GWAS with in-depth phenotyping of affective temperaments, considered as subclinical manifestations and highrisk states for affective disorders, in a general population sample of European origin. Affective temperaments were measured by TEMPS-A. SNP-level association was assessed by linear regression models, assuming an additive genetic effect, using PLINK1.9. Gender, age, the first ten principal components (PCs) and the other four temperaments were included in the regression models as covariates. SNP-level relevances ( $p$-values) were aggregated to gene level using the PEGASUS method ${ }^{1}$. In SNP-based tests, a Bonferroni-corrected significance threshold of $p \leq 5.0 \times 10^{-8}$ and a suggestive significance threshold of $p \leq 1.0 \times 10^{-5}$, whereas in gene-based tests a Bonferroni-corrected significance of $2.0 \times 10^{-6}$ and a suggestive significance of $p \leq 4.0 \times 10^{-4}$ was established. To explore known functional effects of the most significant SNPs, FUMA v1.3.5 was used. We identified 1 significant and 21 suggestively significant SNPs in ADGRB3, expressed in the brain, for anxious temperament. Several other brain-relevant SNPs and genes emerged at suggestive significance for the other temperaments. Functional analyses reflecting effect on gene expression and participation in chromatin interactions also pointed to several genes expressed in the brain with potentially relevant phenotypes regulated by our top SNPs. Our findings need to be tested in larger GWA studies and candidate gene analyses in well-phenotyped samples in relation to affective disorders and related phenotypes.
\end{abstract}

\section{Introduction}

Depression is a severe illness causing significant dysfunction and suffering. Unlike other illnesses where there is significant new progress and paradigm shifts in therapy also due to understanding and exploiting genetic variation associated with the illness, there is, with the exception of

\footnotetext{
Correspondence: Xenia Gonda (gonda.xenia@med.semmelweis-univ.hu) ${ }^{1}$ NAP-2-SE New Antidepressant Target Research Group, Hungarian Brain Research Program, Semmelweis University, Budapest, Hungary ${ }^{2}$ Department of Psychiatry and Psychotherapy, Faculty of Medicine, Semmelweis University, Budapest, Hungary

Full list of author information is available at the end of the article

These authors contributed equally: Xenia Gonda, Nora Eszlari
}

the promising introduction of glutamatergic approaches, a void of new approaches to understanding and treatment of affective disorders contributing to depression predicted to be the illness associated with the highest disease burden in the next very few years ${ }^{2}$.

One reason for our lack of sufficient insight into the etiopathology and neurobiology of depression is the failure to identify replicable genetic variation associated with the emergence and clinical characteristics of this illness. Although candidate gene studies targeted a large number of possible variants, only a few of these were investigated in at least three studies and even less were replicated ${ }^{3}$.

\section{(c) The Author(s) 2021}

(c) (i) Open Access This article is licensed under a Creative Commons Attribution 4.0 International License, which permits use, sharing, adaptation, distribution and reproduction cc. in any medium or format, as long as you give appropriate credit to the original author(s) and the source, provide a link to the Creative Commons license, and indicate if changes were made. The images or other third party material in this article are included in the article's Creative Commons license, unless indicated otherwise in a credit line to the material. If material is not included in the article's Creative Commons license and your intended use is not permitted by statutory regulation or exceeds the permitted use, you will need to obtain permission directly from the copyright holder. To view a copy of this license, visit http://creativecommons.org/licenses/by/4.0/. 
Genome-wide analysis approaches similarly yielded less results in case of depression compared to other psychiatric illnesses and, where suggestive significant results were retrieved, these did not confirm the role of previous candidate genes, nor could they be replicated in subsequent genome-wide association studies (GWASs). However, the most recent large-sample GWAS attempts involving joint databases and megasamples at the sacrifice of accurate phenotyping managed to identify a few significant variants with 102 significant hits in the latest study ${ }^{4}$.

However, the lack of in-depth and precise phenotyping may be a crucial weakness of such studies, as depression as a disease category is a highly heterogeneous phenomenon, where divergent clinical manifestations may equally be labelled as depressive disorder without a single overlapping symptom ${ }^{5}$, and in the background of such heterogeneous clinical symptoms divergent neurobiological pathways and distinct genetic variation are likely to play a role $^{6}$. Subtypes of depression with clinically highly distinct features, such as unipolar or bipolar depression ${ }^{7}$, alexythmic depression ${ }^{8}$, melancholic and atypical depression $^{9,10}$, or depression associated with increased suicidal risk $^{10}$ to name only a few, require focusing on different psychosocial or neurobiological targets, and for this, understanding their distinctive genetic determinants would provide the missing background and first step. Given the lack of proper efficacy of current, monoaminebased treatment approaches to depression, not only new pharmacological targets would be needed but novel agents need to be matched to more clear-cut illness phenotypes within the heterogeneous depression group ${ }^{6}$.

One possible approach to understanding depression via a more homogeneous categorization is the use of endophenotypes related to mood disorders, which are, by definition, better characterized and can be more closely mapped to singular neurobiological alterations ${ }^{11}$. Affective temperaments, thought to possess a strong biological background, manifesting in an early age and persisting through the life span, and being closely linked to affective disorders constituting in their more dominant manifestation a high-risk state or the subclinical form of affective disorders, and possessing a strong pathoplastic role, offer themselves as possible endophenotypes ${ }^{12,13}$. Previous research indicated their genetic associations, strong heritability, more marked manifestation not only in affective disorder patients but also in their first-degree relatives, dimensional distribution in the population, and a strong association with several outcomes of affective illness and general illness course ${ }^{13}$, which argue for using them in GWASs to bring us closer to the identification of genes in depression with an in-depth and precise endophenotyping. Yet, previously, no wholegenome-wide analysis was performed for affective temperaments in a general population sample and there has been only one GWAS in a bipolar patient sample ${ }^{14}$.
The aim of our present study was to perform a pilot GWAS on affective temperaments in a European general population sample.

\section{Methods \\ Participants}

The study was part of the NewMood project (New Molecules in Mood Disorders, Sixth Framework Program of the EU, LSHM-CT-2004-503474) funded by the European Union. NewMood has been a collaboration between 13 clinical and basic science research groups, across 10 European countries aimed at identifying new molecules in the background of mood disorders (www. newmood.co.uk), focusing on genetics with the specific aim of demonstrating allelic variation in association with vulnerability markers of depression, translation from rodent to human models, and specifically using translational endophenotypic markers rather than symptoms or diagnoses ${ }^{15-17}$. In the human clinical part of the project over 3000 participants of European White ethnic origin aged 18-60 years, in Greater Manchester and Budapest, were recruited via advertisements, general practices and a website. Participants filled out a questionnaire pack for precise and in-depth phenotyping of multiple endophenotypes related to susceptibility to depression, provided detailed background information on personal and family psychiatric history, as well as demographic and socioeconomic circumstances, and provided DNA using a saliva sampling kit. In the present study, we used phenotypic and genotypic data from 775 participants in the Budapest cohort. All procedures were carried out in accordance with the Declaration of Helsinki and were approved by the Scientific and Research Ethics Committee of the Medical Research Council, Budapest, Hungary. All participants provided a written informed consent prior to participating in the study.

\section{Phenotype}

Participants filled out the NewMood questionnaire pack, including the standardized Hungarian version of the 110-item Temperament Evaluation of Memphis, Pisa, Paris and San Diego (TEMPS-A) questionnaire to measure affective temperaments ${ }^{12,18}$ and a background questionnaire including questions on age, gender, previous psychiatric illness, family history of psychiatric illness, somatic disorders and relevant demographic information. The TEMPS-A consists of five scales measuring the five affective temperaments described by Akiskal based on a clinical population and their first-degree relatives, including the depressive, cyclothymic, hyperthymic, irritable and anxious temperaments. Each affective temperament score was calculated as a continuous weighted score by dividing the sum of item scores by the number of completed items. 


\section{Genotyping, quality control and imputation}

Participants provided DNA by a genetic saliva sampling kit. Genomic DNA was extracted from buccal mucosa cells according to established protocols ${ }^{19}$. Genotyping was performed using Illumina's CoreExom PsychChip, yielding a total of 573,141 variants, the genomic positions of which were defined according to the build GRCh37/hg19. Quality control and imputation was based on refs. ${ }^{20,21}$ (see Supplementary File 1).

\section{Statistical analyses}

Descriptive statistics were done with SPSS25.

Primary single-nucleotide polymorphism (SNP)-based association tests for each affective temperament phenotype were calculated using linear regression models in Plink 1.9 (https://www.cog-genomics.org/plink2), assuming an additive genetic effect. All models contained the first ten calculated principal components (PCs), gender, age, and the other four temperaments as covariates.

Gene-based tests for each affective temperament phenotype were calculated using Pegasus ${ }^{1}$. Significance $(p-$ value) of genes is calculated by a method that aggregates variant-level $p$-values and takes into account the dependence between them (induced by the linkage between the respective variants) by applying a null model of a multivariate normal distribution with a covariance matrix reflecting the aforementioned linkage.

To explore the known functional effects of our most significant SNPs as reported in public open databases based on expression quantitative trait loci (eQTL) and three-dimensional chromatin interaction, we used FUMA v1.3.5. $5^{22}$, with a $p \leq 1 \times 10^{-5}$ threshold for lead SNPs, an $R^{2} \geq 0.5$ to define a genomic risk locus around a lead SNP, and a $p \leq 0.05$ to involve SNPs into it. Each SNP of the genomic risk loci (referred to as top SNPs or our most significant SNPs) were mapped to a gene if either residing within gene boundaries extended by 10,000 base pairs, or having a false discovery rate $q \leq 0.05$ with it in the external eQTL, or a $q \leq 1 \times 10^{-6}$ with its promoter region in the external chromatin interaction dataset ${ }^{22}$.

\section{Results}

\section{Characteristics of the sample}

Imputation and quality-control steps yielded 2,550,710 SNPs and 775 subjects with data on the 5 affective temperaments, gender, age, and quality-controlled genomic data. By convention the threshold for statistical significance is based on a Bonferroni correction for 1 million comparisons, yielding a threshold of $p \leq 5.0 \times 10^{-8}$, whereas at the SNP-level $p \leq 1.0 \times 10^{-5}$ was determined as threshold for suggestive significance ${ }^{23}$. For gene-based tests, a Bonferroni-corrected significance threshold of $2.0 \times 10^{-6}$ was applied and, at the gene level, $p \leq 4.0 \times 10^{-4}$ was determined as threshold for suggestive significance, the former one corresponding to the Bonferroni-corrected nominal significance level of 0.05 assuming 25,000 independent tests (the approximate number of the genes tested) and the latter one corresponding to that number multiplied by 200 (as in the case of variant-level tests).

Descriptive statistics on affective temperament scores, gender, age, self-reported psychiatric history and treatment are provided in Supplementary Table S1. Affective temperament scales were significantly and at least moderately correlated with each other in our sample (Supplementary Table S2); thus, when analysing specific variability of each affective temperament scale, the other four temperament scales were covariates in the model.

\section{SNPs in the background of the five affective temperaments Significant and suggestively significant findings in SNP- based tests for anxious temperament}

With respect to specific SNPs, SNP-based association tests yielded a genomic inflation estimate (based on median $\chi^{2}$ ) of $\lambda=1.00703$ for anxious temperament. For the quantile-quantile (QQ) plot, see Supplementary Fig. S1. In case of anxious temperament, rs3798978 within the $A D G R B 3$ gene survived correction for genome-wide significance $\left(p=4.44 \times 10^{-8}\right)$, on chromosome 6 , whereas 21 other SNPs within ADGRB3 (Fig. 1A) and 7 other SNPs in intergenic regions on chromosome 8,11 , and 17 had a suggestive significance after correction (Fig. 2 I-A and Table 1).

\section{Suggestively significant findings in SNP-based tests for cyclothymic temperament}

For cyclothymic temperament, $\lambda$-value resulting from genome-wide SNP-based tests was $\lambda=1.00000$. For the QQ plot, see Supplementary Fig. S2. No SNP survived Bonferroni correction for multiple testing; however, 12 SNPs showed a suggestive significance, 4 of which reside in ERBB4 (Fig. 1B), whereas the others reside in genes CSMD1, NAP1L4, SNORA54 or are intergenic (Fig. 2 I-B and Table 1).

\section{Suggestively significant findings in SNP-based tests for depressive temperament}

In case of depressive temperament, genome-wide SNPbased tests yielded a genomic inflation factor of $\lambda=$ 1.00172. For the QQ plot, see Supplementary Fig. S3. No SNP survived Bonferroni correction for multiple testing, but five SNPs showed a suggestive significance, one of which resides in the $S G C Z$ gene, whereas the rest are intergenic (Fig. $2 \mathrm{I}-\mathrm{C}$ and Table 1).

\section{Suggestively significant findings in SNP-based tests for irritable temperament}

In case of irritable temperament, genome-wide SNPbased tests yielded a genomic inflation factor of $\lambda=$ 1.00000. For the QQ plot, see Supplementary Fig. S4. No 


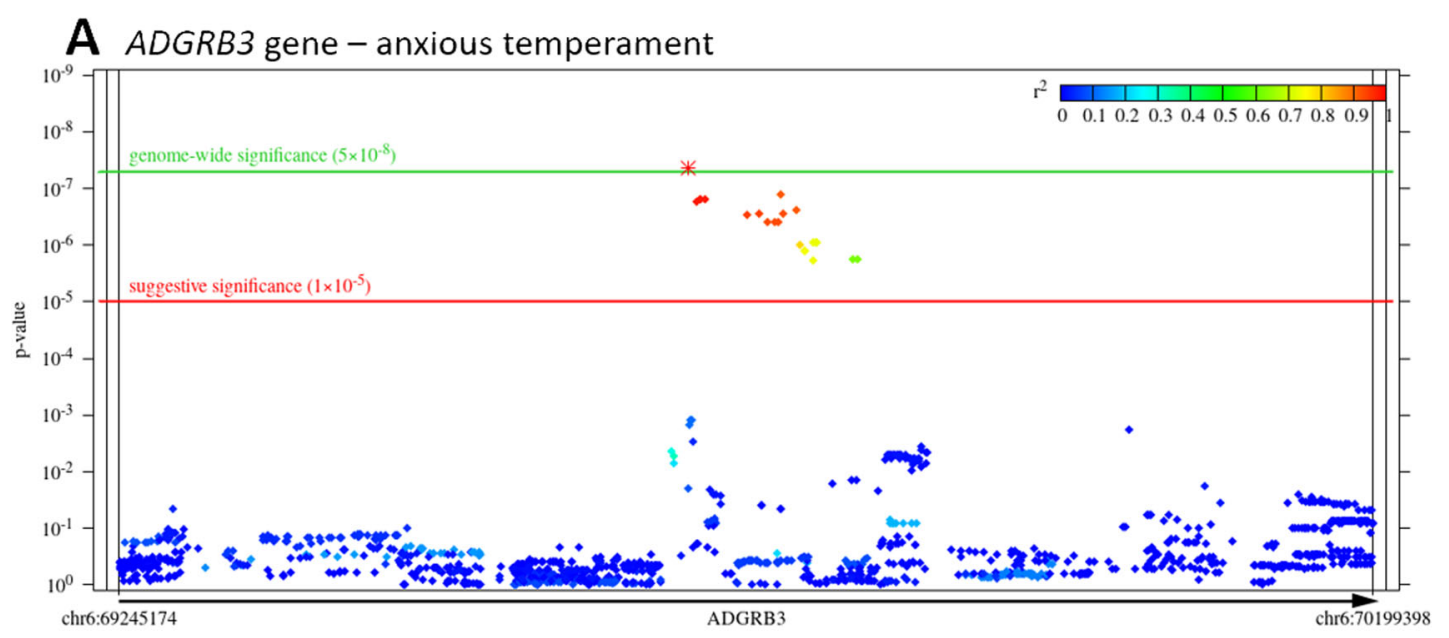

B ERBB4 gene-cyclothymic temperament

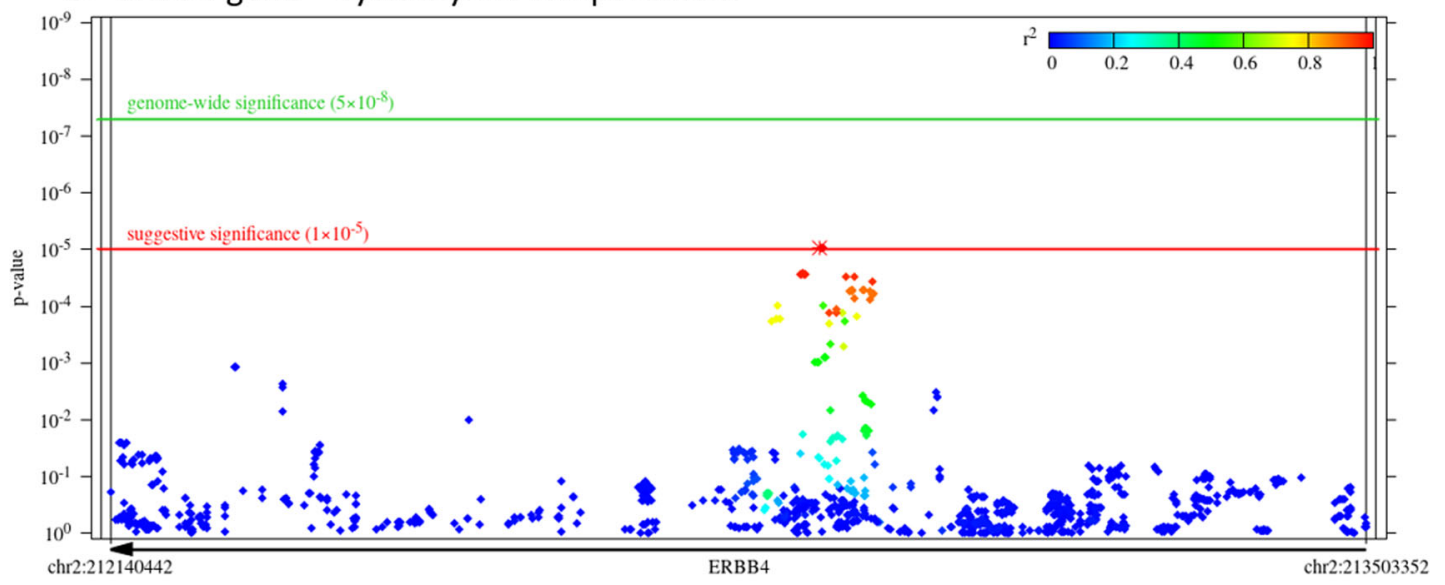

C SHISA6 gene - irritable temperament

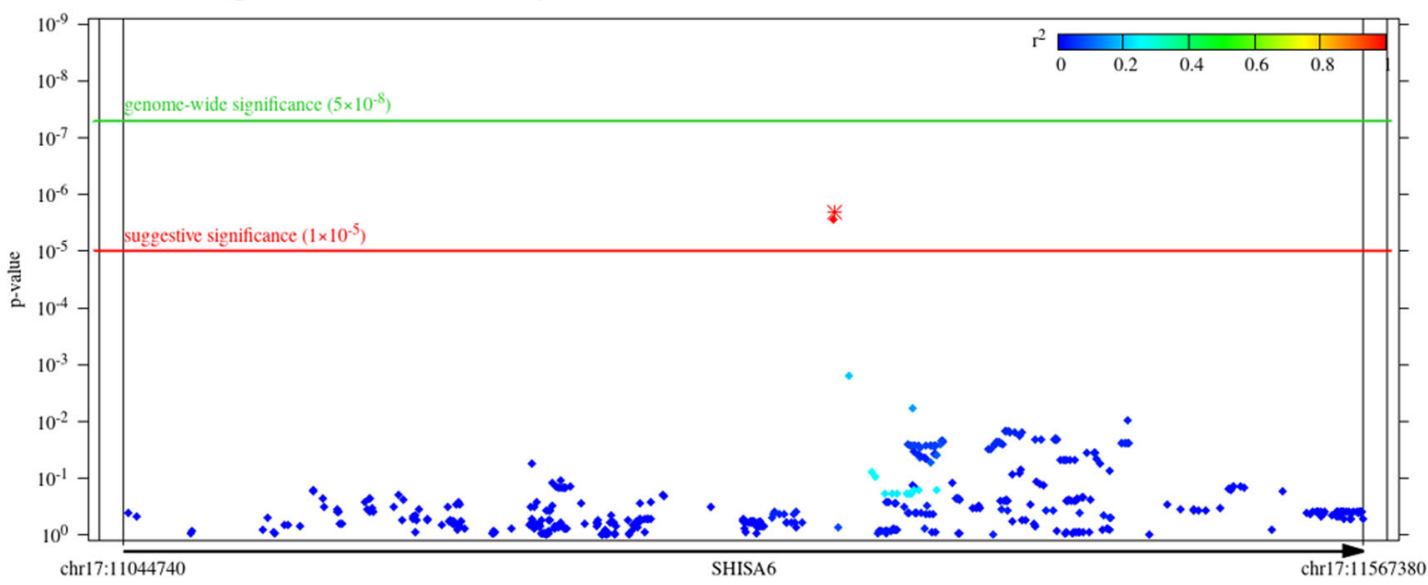

Fig. 1 Zoomed Manhattan plots of significant or suggestively significant SNPs for anxious, cyclothymic and irritable temperaments.

Zoomed Manhattan plots of A ADGRB3 gene on chromosome 6 for anxious temperament as the outcome phenotype (rs3798978 survived correction for genome-wide significance $\left(p=4.44 \times 10^{-8}\right.$ ), whereas 21 other SNPs showed suggestive significance); $\mathbf{B}$ ERBB4 gene on chromosome 2 for cyclothymic temperament as the outcome phenotype (4 SNPs showed suggestive significance); and C SHISA6 gene on chromosome 17 for irritable temperament as the outcome phenotype (2 SNPs showed suggestive significance). P-value is displayed in function of genomic position for each single-nucleotide polymorphism (SNP) in the region. Colours denote the $r^{2}$-value of linkage disequilibrium (LD) with the most significant SNP (marked with asterisk). Gene boundaries and their extension by 10,000 base pairs (as defined for the gene-based tests) are marked with vertical lines. 


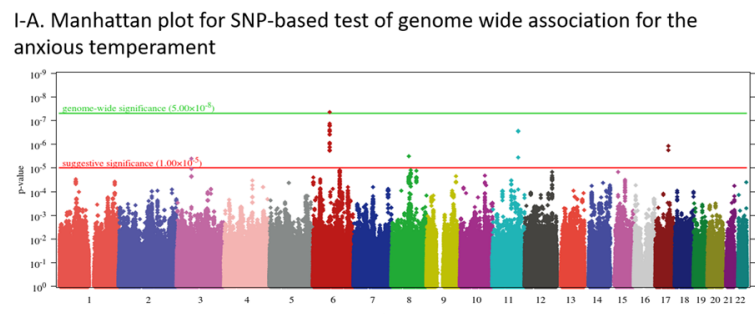

I-B. Manhattan plot for SNP-based test of genome wide association for the cyclothymic temperament

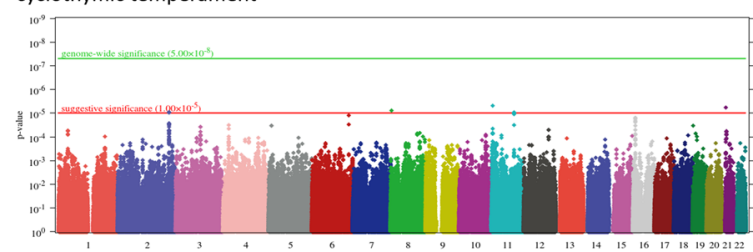

I-C. Manhattan plot for SNP-based test of genome wide association for the depressive temperament

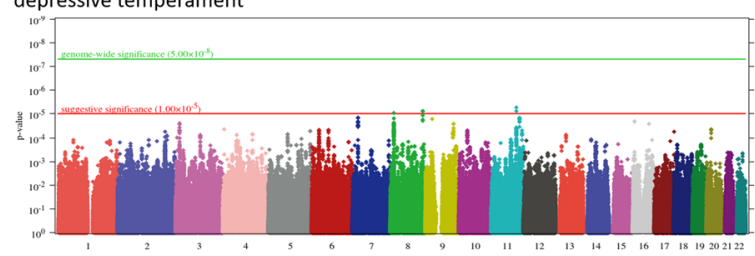

I-D. Manhattan plot for SNP-based test of genome wide association for the irritable temperament

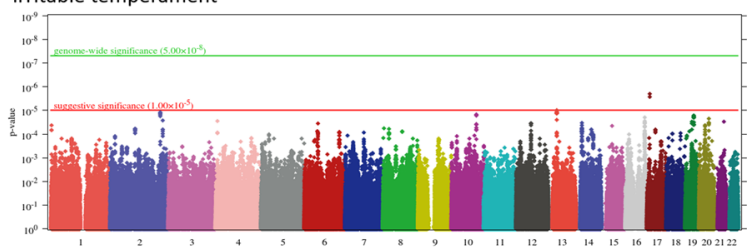

I-E. Manhattan plot for SNP-based test of genome wide association for the hyperthymic temperament

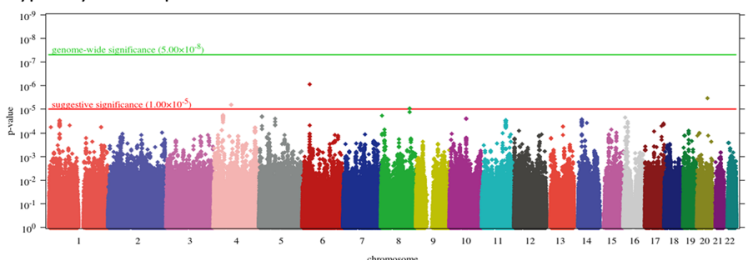

II-A. Manhattan plot for gene-based test of genome wide association for the anxious temperament

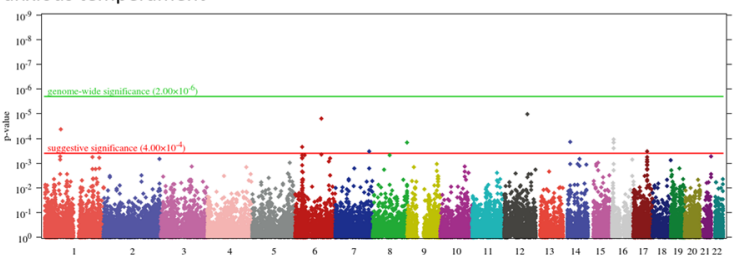

II-B. Manhattan plot for gene-based test of genome wide association for the cyclothymic temperament

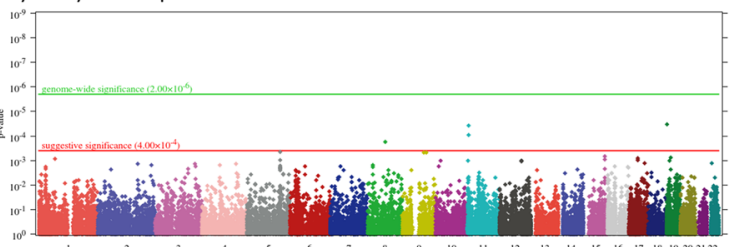

II-C. Manhattan plot for gene-based test of genome wide association for the depressive temperament

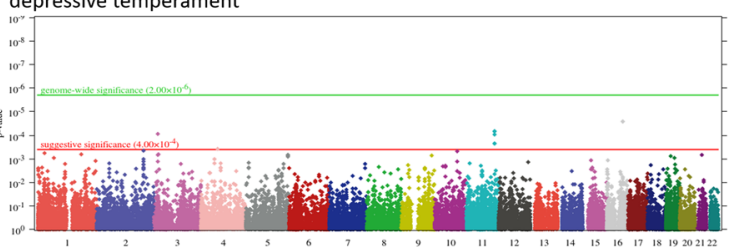

II-D. Manhattan plot for gene-based test of genome wide association for the irritable temperament

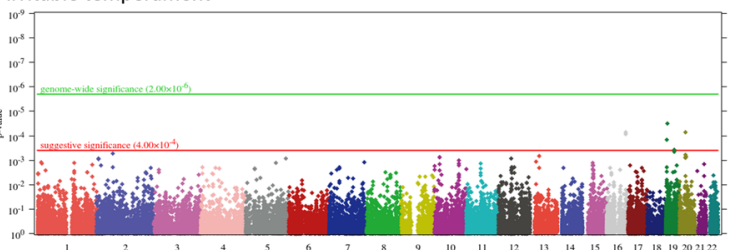

II-E. Manhattan plot for gene-based test of genome wide association for the hyperthymic temperament

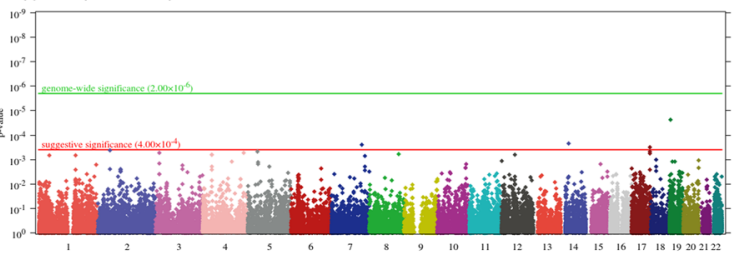

Fig. 2 Manhattan plots of genome-wide SNP-based and gene-based tests for the five affective temperaments. Manhattan plots of genomewide SNP-based tests (I) and gene-based tests (II) for anxious (I-A, II-A), depressive (I-B, II-B), cyclothymic (I-C, II-C), irritable (I-D, II-D) and hyperthymic (I-E, II-E) temperaments as outcome. P-value is displayed in function of genomic position for each single-nucleotide polymorphism (SNP). The red and green lines denote the levels of a suggestive and a genome-wide significance, respectively. P-value is displayed in function of genomic position for each single-nucleotide polymorphism (SNP). The red and green lines denote the levels of a suggestive and a genome-wide significance, respectively.

SNP survived correction for multiple testing; however, three SNPs showed a suggestive significance (Fig. 2 I-D and Table 1). Except for one intergenic SNP, the other two SNPs are mapped to the SHISA6 gene (Fig. 1C).

\section{Suggestively significant findings in SNP-based tests for hyperthymic temperament}

In case of hyperthymic temperament, genome-wide SNP-based tests yielded a genomic inflation factor of $\lambda=$ 1.02018. For the QQ plot, see Supplementary Fig. S5. No
SNP survived Bonferroni correction for multiple testing, but five SNPs showed a suggestive significance that either reside in genes CDSN, PSORS1C1, or are intergenic (Fig. 2 I-E and Table 1).

\section{Suggestively significant findings in gene-based tests for affective temperament phenotypes}

Hits with genome-wide suggestive $p$-values at the gene level for each temperament together with the most significant SNP within each gene are shown in Table 2 and 
Table 1 Most significant SNPs with genome-wide or suggestive significances for the five affective temperaments.

\begin{tabular}{|c|c|c|c|c|c|c|}
\hline Chromosome & SNP & Base position & Minor/effect allele & $\beta$ & $P$ & Genes \\
\hline \multicolumn{7}{|c|}{ Anxious temperament } \\
\hline 3 & rs1281465 & $59,664,687$ & G & 0.03152 & $4.03 E-06$ & LOC339902 \\
\hline 6 & rs3798978 & $69,678,213$ & G & 0.08709 & $4.44 \mathrm{E}-08$ & ADGRB3 \\
\hline 6 & rs34526480 & $69,684,542$ & G & 0.08332 & $1.73 E-07$ & ADGRB3 \\
\hline 6 & rs13191706 & $69,686,817$ & G & 0.08362 & $1.58 \mathrm{E}-07$ & ADGRB3 \\
\hline 6 & rs13194716 & $69,686,889$ & G & 0.08362 & $1.58 \mathrm{E}-07$ & ADGRB3 \\
\hline 6 & rs71555397 & $69,690,915$ & G & 0.08362 & $1.58 \mathrm{E}-07$ & ADGRB3 \\
\hline 6 & rs3799007 & $69,723,045$ & $C$ & 0.08099 & $2.85 E-07$ & ADGRB3 \\
\hline 6 & rs117667441 & $69,731,898$ & $\mathrm{~T}$ & 0.08154 & $2.76 \mathrm{E}-07$ & ADGRB3 \\
\hline 6 & rs80164607 & $69,737,887$ & $\mathrm{~T}$ & 0.07958 & $3.95 E-07$ & ADGRB3 \\
\hline 6 & rs79911876 & $69,743,496$ & $\mathrm{~T}$ & 0.07958 & $3.95 E-07$ & ADGRB3 \\
\hline 6 & rs75556966 & $69,746,343$ & $\mathrm{~T}$ & 0.07958 & $3.95 \mathrm{E}-07$ & ADGRB3 \\
\hline 6 & rs74802157 & $69,748,723$ & $\mathrm{~T}$ & 0.08445 & $1.30 E-07$ & ADGRB3 \\
\hline 6 & rs118099456 & $69,750,540$ & G & 0.08279 & $2.71 E-07$ & ADGRB3 \\
\hline 6 & rs3799021 & $69,760,328$ & G & 0.08314 & $2.41 \mathrm{E}-07$ & ADGRB3 \\
\hline 6 & rs62416781 & $69,762,935$ & A & 0.07648 & $9.92 \mathrm{E}-07$ & ADGRB3 \\
\hline 6 & rs62416782 & $69,766,653$ & A & 0.07518 & $1.28 \mathrm{E}-06$ & ADGRB3 \\
\hline 6 & rs62416803 & $69,766,783$ & $\mathrm{~T}$ & 0.07518 & $1.28 \mathrm{E}-06$ & ADGRB3 \\
\hline 6 & rs3734465 & $69,772,650$ & G & 0.07542 & $8.95 E-07$ & ADGRB3 \\
\hline 6 & rs62416807 & $69,773,007$ & G & 0.07437 & $1.88 \mathrm{E}-06$ & ADGRB3 \\
\hline 6 & rs3799023 & $69,774,810$ & C & 0.07542 & $8.95 \mathrm{E}-07$ & ADGRB3 \\
\hline 6 & rs79811527 & $69,776,038$ & $\mathrm{~T}$ & 0.07542 & $8.95 \mathrm{E}-07$ & ADGRB3 \\
\hline 6 & rs3799030 & $69,803,044$ & A & 0.07696 & $1.81 E-06$ & ADGRB3 \\
\hline 6 & rs62406773 & $69,806,494$ & $\mathrm{~T}$ & 0.0774 & $1.75 E-06$ & ADGRB3 \\
\hline 8 & rs13251367 & $72,001,931$ & A & 0.03391 & $3.26 \mathrm{E}-06$ & XKR9 || EYA1 \\
\hline 11 & rs484334 & $107,769,407$ & C & -0.03474 & $2.90 E-07$ & SLC35F2 || LOC643949 \\
\hline 11 & rs563811 & $107,772,104$ & A & -0.0347 & $2.72 \mathrm{E}-07$ & SLC35F2 || LOC643949 \\
\hline 11 & rs552905 & $107,777,210$ & A & -0.03163 & $3.55 E-06$ & SLC35F2 || LOC643949 \\
\hline 17 & rs7226241 & $53,573,452$ & A & 0.05233 & $1.20 E-06$ & MMD || TMEM100 \\
\hline 17 & rs1553677 & $53,573,902$ & A & 0.05127 & $1.77 E-06$ & MMD || TMEM100 \\
\hline \multicolumn{7}{|c|}{ Cyclothymic temperament } \\
\hline 2 & rs73988952 & $212,909,118$ & A & -0.05511 & $9.17 E-06$ & ERBB4 \\
\hline 2 & rs73988954 & $212,911,142$ & C & -0.05511 & $9.17 E-06$ & ERBB4 \\
\hline 2 & rs141189957 & $212,912,423$ & C & -0.05511 & $9.17 E-06$ & ERBB4 \\
\hline 2 & rs10445735 & $212,913,183$ & G & -0.05511 & $9.17 E-06$ & ERBB4 \\
\hline 8 & rs2656285 & $4,117,538$ & A & -0.03344 & $7.80 E-06$ & CSMD1 \\
\hline 11 & rs7948848: & $2,981,896$ & A & -0.03195 & $4.72 E-06$ & NAP1L4. SNORA54 \\
\hline 11 & rs11020416 & $93,293,727$ & C & 0.03278 & $9.79 E-06$ & LOC729466 || LOC642897 \\
\hline 11 & rs2895473 & $93,293,780$ & A & 0.03273 & $9.81 \mathrm{E}-06$ & LOC729466 || LOC642897 \\
\hline
\end{tabular}


Table 1 continued

\begin{tabular}{|c|c|c|c|c|c|c|}
\hline Chromosome & SNP & Base position & Minor/effect allele & $\beta$ & $P$ & Genes \\
\hline 11 & rs4753475 & $93,295,861$ & A & 0.03274 & $9.86 \mathrm{E}-06$ & LOC729466 || LOC642897 \\
\hline 11 & rs60944979 & $93,297,230$ & $C$ & 0.03295 & $8.93 E-06$ & LOC729466 || LOC642897 \\
\hline 11 & rs4753086:93297625 & $93,297,625$ & C & 0.03284 & $9.61 E-06$ & LOC729466 || LOC642897 \\
\hline 21 & rs2823289 & $16,841,195$ & G & 0.03159 & $5.70 E-06$ & NRIP1 || CYCSP42 \\
\hline \multicolumn{7}{|c|}{ Depressive temperament } \\
\hline 8 & rs34835594 & $14,570,438$ & A & 0.02743 & $9.14 \mathrm{E}-06$ & SGCZ \\
\hline 8 & rs7831625 & $135,076,495$ & $\mathrm{~T}$ & 0.04273 & $7.56 \mathrm{E}-06$ & LOC100129104 || ZFAT \\
\hline 8 & rs7836140 & $135,077,157$ & A & 0.04273 & $7.56 \mathrm{E}-06$ & LOC100129104 || ZFAT \\
\hline 8 & rs16905065 & $135,077,484$ & G & 0.04273 & $7.56 \mathrm{E}-06$ & LOC100129104 || ZFAT \\
\hline 11 & rs1917447 & $103,498,125$ & C & -0.02727 & $7.56 \mathrm{E}-06$ & DYNC2H1 || PDGFD \\
\hline 11 & rs1917448 & $103,498,150$ & G & -0.02761 & $5.26 \mathrm{E}-06$ & DYNC2H1 \| PDGFD \\
\hline \multicolumn{7}{|c|}{ Irritable temperament } \\
\hline 13 & rs9542430 & $35,400,570$ & $\mathrm{~T}$ & 0.03006 & $9.73 E-06$ & LOC100129452 || NBEA \\
\hline 17 & rs3111836 & $11,344,043$ & G & 0.03776 & $2.74 E-06$ & SHISA6 \\
\hline 17 & rs2969184 & $11,344,615$ & A & 0.03824 & $2.02 E-06$ & SHISA6 \\
\hline \multicolumn{7}{|c|}{ Hyperthymic temperament } \\
\hline 4 & rs2123430 & $71,999,712$ & A & 0.04798 & $6.51 E-06$ & LOC100128311 || LOC727995 \\
\hline 4 & rs11729471 & $72,000,800$ & A & 0.04829 & $6.40 E-06$ & LOC100128311 || LOC727995 \\
\hline 6 & rs3131004 & $31,095,294$ & G & -0.04087 & $9.00 \mathrm{E}-07$ & CDSN.PSORSIC1 \\
\hline 8 & rs1793704 & $118,307,005$ & $\mathrm{~T}$ & 0.03791 & $9.23 E-06$ & SLC30A8 || MED3O \\
\hline 20 & rs75945142 & $41,850,477$ & A & -0.0455 & $3.31 E-06$ & \\
\hline
\end{tabular}

$p$-value; Bonferroni-corrected $p$-level for significance: $p \leq 5 \times 10^{-8}$. P-level of suggestive significance: $p \leq 1 \times 10^{-5}$. Significant hits are marked with bold.

Fig. 2-II. No genes reached genome-wide significance after correction for multiple testing in case of any of the temperaments; however, several genes with suggestive significance were identified for each affective temperament.

\section{Suggestively significant findings in gene-based tests for anxious temperament}

In case of anxious temperament, for the QQ plot, see Supplementary Fig. S6; INSL5, FYN, HCG22, PIP, MIR1251, HECTD1, ROGDI, SEPT12, SMIM22 and MIR454 genes survived the threshold for suggestive significance after correction for multiple testing (Table 2 and Fig. 2 II-A).

\section{Suggestively significant findings in gene-based tests for cyclothymic temperament}

In case of cyclothymic temperament, for the QQ plot, see Supplementary Fig. S7; C8orf89, NAP1L4 and SNORA54 genes survived the threshold for suggestive significance after correction for multiple testing (Table 2 and Fig. 2 II-B).
Suggestively significant findings in gene-based tests for depressive temperament

In case of depressive temperament, for the QQ plot, see Supplementary Fig. S8; after correction for multiple testing, MIR4270, UGT2B7, LOC100652768, PCSK7, SIDT2 and $T A G L N$ genes remained suggestively significant (Table 2 and Fig. 2 II-C).

\section{Suggestively significant findings in gene-based tests for irritable temperament}

In case of irritable temperament, for the QQ plot, see Supplementary Fig. S9; CENPN, CMC2, MBD3L3, ZNF566, ZNF77 and ENTPD6 genes remained suggestively significant after correction for multiple testing (Table 2 and Fig. 2 II-D).

Suggestively significant findings in gene-based tests for hyperthymic temperament

In case of hyperthymic temperament, for the QQ plot, see Supplementary Fig. S10; LOC100506682, SNX6, CANT1 and ZNF77 were suggestively significant after correction for multiple testing (Table 2 and Fig. 2 II-E). 
Table 2 Genes with genome-wide suggestive significance and most significant SNPs for the five affective temperaments.

\begin{tabular}{|c|c|c|c|c|}
\hline Gene & $p$ & No. of SNPs & Best SNP & SNP $p$-value \\
\hline \multicolumn{5}{|c|}{ Anxious temperament } \\
\hline INSL5 & $4.23 E-05$ & 13 & rs1353716 & $3.05 E-05$ \\
\hline FYN & $1.60 E-05$ & 225 & rs1409839 & $1.22 \mathrm{E}-05$ \\
\hline HCG22 & 0.00021981 & 194 & rs2905757 & $7.83 E-05$ \\
\hline PIP & 0.00032626 & 27 & rs10270005 & $8.70 E-05$ \\
\hline MIR1251 & $1.02 E-05$ & 7 & rs4418855 & 0.0001149 \\
\hline HECTD1 & 0.000138739 & 49 & rs11625570 & $5.74 \mathrm{E}-05$ \\
\hline ROGDI & 0.000245947 & 5 & rs9673735 & 0.0001447 \\
\hline SEPT12 & 0.000105394 & 6 & rs4389143 & $5.22 E-05$ \\
\hline SMIM22 & 0.00014637 & 8 & rs4389143 & $5.22 \mathrm{E}-05$ \\
\hline MIR454 & 0.000333644 & 10 & rs8077052 & 0.0003431 \\
\hline \multicolumn{5}{|c|}{ Cyclothymic temperament } \\
\hline C8orf89 & 0.000172857 & 8 & rs2925445 & 0.0001995 \\
\hline NAPIL4 & $9.29 E-05$ & 107 & rs7948848 & $4.72 E-06$ \\
\hline SNORA54 & $3.75 E-05$ & 46 & rs7948848 & $4.72 E-06$ \\
\hline \multicolumn{5}{|c|}{ Depressive temperament } \\
\hline MIR4270 & $8.68 \mathrm{E}-05$ & 30 & rs9821793 & $3.73 E-05$ \\
\hline UGT2B7 & 0.000363155 & 345 & rs4694604 & 0.0002398 \\
\hline LOC100652768 & $9.10 E-05$ & 39 & rs634960 & $1.43 E-05$ \\
\hline PCSK7 & 0.000214682 & 53 & rs634960 & $1.43 E-05$ \\
\hline SIDT2 & 0.000214728 & 46 & rs634960 & $1.43 E-05$ \\
\hline TAGLN & $6.61 E-05$ & 42 & rs634960 & $1.43 E-05$ \\
\hline \multicolumn{5}{|c|}{ Irritable temperament } \\
\hline CENPN & $7.23 E-05$ & 50 & rs2602428 & $1.97 E-05$ \\
\hline CMC2 & $8.50 E-05$ & 71 & rs7201499 & $2.76 \mathrm{E}-05$ \\
\hline$M B D 3 L 3$ & $3.05 E-05$ & 2 & rs2967642 & 0.000118 \\
\hline ZNF566 & 0.000364777 & 125 & rs2385795 & $1.75 E-05$ \\
\hline ZNF77 & 0.000148404 & 2 & rs11666431 & 0.0001534 \\
\hline ENTPD6 & $7.07 E-05$ & 54 & rs6115093 & $4.27 E-05$ \\
\hline \multicolumn{5}{|c|}{ Hyperthymic temperament } \\
\hline LOC100506682 & 0.000247609 & 65 & rs10278458 & 0.0002187 \\
\hline SNX6 & 0.00022356 & 55 & rs28421851 & $2.67 E-05$ \\
\hline CANT1 & 0.000306978 & 14 & rs12450407 & $3.98 \mathrm{E}-05$ \\
\hline ZNF77 & $2.31 E-05$ & 2 & rs11666431 & 0.0001303 \\
\hline
\end{tabular}

$p$-value: empirical $p$-value based on one million tests; Bonferroni-corrected $p$ level for significance $p \leq 2 \times 10^{-6}$. Suggestive level of significance $p \leq 4 \times 10^{-4}$.

\section{Genomic location of top SNPs for affective temperament phenotypes}

Proportion of intronic SNPs was nominally significant in case of all five affective temperament phenotypes, and with the exception of anxious temperament phenotype, in case of the other four temperament phenotypes proportion of noncoding RNA intronic SNPs was also nominally significant (Fig. 3). In case of all affective temperament phenotypes, proportion of intronic SNPs, and, with the exception of anxious temperament, proportion of noncoding RNA intronic SNPs were significant, thus, the majority of identified SNPs likely influence function of protein- or RNA-coding genes by mechanisms other than amino acid replacement.

\section{Functional effects of the top SNPs identified for affective} temperament phenotypes on gene expression regulation in the brain

We carried out functional mapping and annotation concerning genes regulated in all tissues and, as we investigated a psychological phenotype, specifically in the brain, by our top SNPs, based on external chromatin interaction databases and eQTL databases GTEx v6 and $\mathrm{v}^{24,25}$, and BRAINEAC ${ }^{26}$, which comprise several brain regions, as well as $x Q T L S$ erver $^{27}$ and CommonMind Consortium $(\mathrm{CMC})^{28}$ samples, which encompass only the dorsolateral prefrontal cortex (DLPFC). FUMA results in case of the five investigated affective temperament phenotypes concerning regulated genes in all tissues and cell types available are shown in Supplementary Tables S3-S12 and Supplementary Figs. S11-S23. Here we only describe and discuss functional effects of top SNPs in case of each affective temperament phenotypes in the brain; however, effects on all tissue types are shown in Supplementary Tables and Figs.

\section{Functional effects of top SNPs identified for the anxious temperament phenotype}

We found that top SNPs for the anxious temperament phenotype on chromosome 11 were associated with gene expression levels in SLC35F2 in the DLPFC (in CMC samples), in the hypothalamus (in GTEx/v7 samples), in nucleus accumbens basal ganglia (in GTEx/v7 samples) and in the pituitary (in GTEx/v7 samples); in ACAT1 (in GTEx/ v6 samples) and $S L N$ (GTEx/v7 samples) in the cerebellum; and in ELMOD1 in the DLPFC (in CMC samples) (Supplementary Table S1 and Supplementary Fig. S13). Top SNPs for the anxious temperament took part in chromatin interactions in the fetal cortex with Lnc-NPAT-2:ACAT; NPAT: 
A Anxious temperament

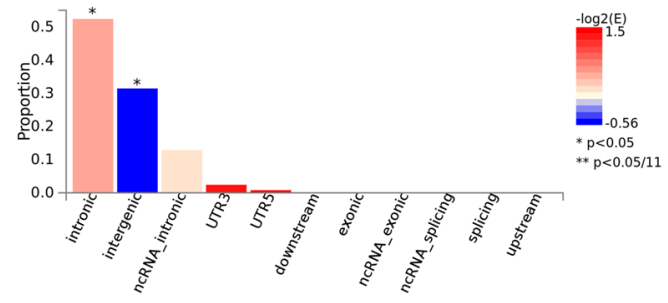

B Cyclothymic temperament

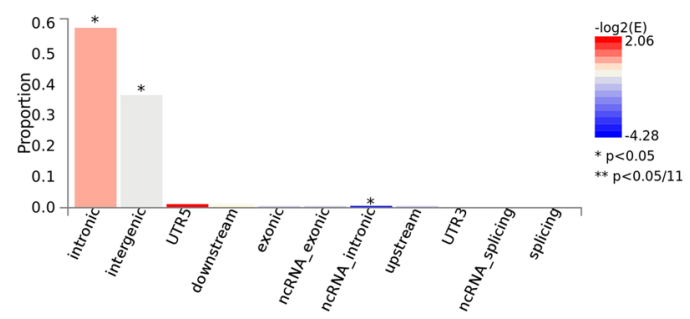

C Depressive temperament

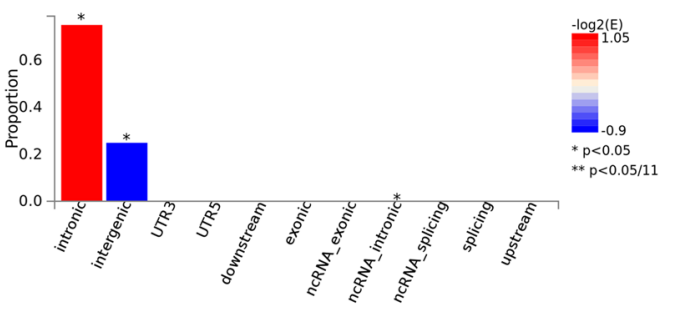

D Irritable temperament

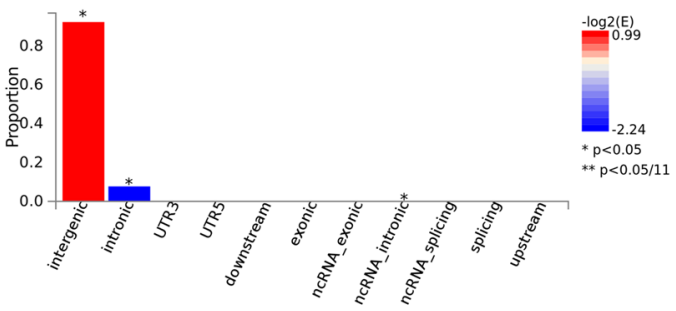

E Hyperthymic temperament

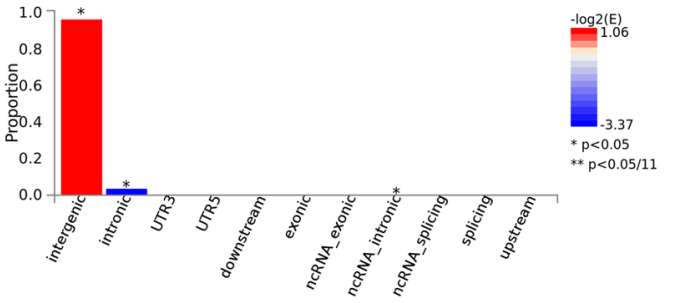

Fig. 3 Genomic location and functional consequences of SNPs for affective temperament phenotypes. Histogram of functional consequences of SNPs for anxious (A), cyclothymic (B), depressive (C), irritable (D), and hyperthymic (E) temperaments.
ATM:Y_RNA; SLN:ENSG00000268602; ENSG00000214306: ENSG00000214305 (Metazoa_SRP) in Giusti-Rodriguez et al. $^{29}$; and in neural progenitor cells with RPL37P15, NPM1P37 and MMD in GS87112 samples (Supplementary Table S4).

Functional effects of top SNPs identified for the cyclothymic temperament phenotype

FUMA has indicated that top SNPs in case of cyclothymic temperament affected expression levels on chromosome 11 in $A C 131971.1$ in the cortex (GTEx/ V7 samples) and expression levels in C11orf54 in the thalamus (BRAINEAC samples), and were associated with expression levels in SLC22A18 (CMC samples), MED17 (CMC samples), NAP1L4 (CMC samples), SCARNA9 (CMC samples and xQTLServer samples) and TAF1D (CMC samples) in the DLPFC (Supplementary Table S5 and Supplementary Fig. S16).

Top SNPs for cyclothymic temperament also took part in chromatin interactions in the adult cortex: RNU1-91P, ZNF195:TSSC2, SRP14P2, SMCO4 in Giusti-Rodriguez et al. $^{29}$ and in neural progenitor cells in GSE87112 database with ERBB4, AC012491.1, AIMP2, SLC36A4: C14orf2; Lnc-DEUP1-1 (Supplementary Table S6).

Functional effects of top SNPs identified for the depressive temperament phenotype

In case of the depressive temperament phenotype, the identified top SNPs did not affect expression levels of genes in the brain (Supplementary Table S7) and did not take part in chromatin interactions with known genes (Supplementary Table S7).

Functional effects of top SNPs identified for the irritable temperament phenotype

Top SNPs associated with the irritable temperament did not influence expression levels of any genes according to FUMA (Supplementary Table S9); however, they took part in chromatin interactions in the adult and fetal cortex with $N B E A^{29}$, and in the fetal cortex with $R F C 3^{29}$ (Supplementary Table S10).

\section{Functional effects of top SNPs identified for the hyperthymic temperament phenotype}

FUMA revealed that top SNPs for hyperthymic temperament influenced expression levels in HCG27 in DLPFC (in CMC) samples in chromosome 6, and in $S L C 4 A 4$ in DLPFC, HLA-C in DLPFC (in CMC samples) and $C 4 A$ in DLPFC (in CMC samples) in chromosome 6 (Supplementary Table S11 and Supplementary Fig. S22). Top SNPs for the hyperthymic temperament were also 
involved in chromatin interactions in neural progenitor cells with EIF3S3:UTP23 in GSE87112 database (Supplementary Table S12).

\section{Discussion}

Our present study is the first to analyse the genomewide association of affective temperaments in a general European population sample with precise in-depth phenotyping for these endophenotypes. As affective temperaments can be considered sub-affective manifestations of, and when present in a dominant form, high-risk states for the development of different types of affective illnesses ${ }^{12}$, they may provide a complex approach to understand the genetic background of different affective disorders and mood syndromes yielding clinical relevance for our present results. Although several suggestively significant SNPs and genes with potential relevance for affective disorders and other psychiatric and psychological phenotypes emerged, we most notably identified one SNP in case of the anxious temperament, which survived correction for multiple testing in $A D G R B 3$ where several other suggestively significant SNPs also underline the potential importance of this gene.

Previously, only one study investigated the genetic background of affective temperaments in a whole-genome analysis approach, in 1263 bipolar patients in the Bipolar Genome Study ${ }^{14}$ identifying 3 genome-wide significant SNPs for the hyperthymic temperament near $M D M I$ on chromosome 12 and FBLNI on chromosome 22, and 2 significant SNPs on chromosome 1 within neighbouring INTS7 and DTL genes, which led the authors to conclude that temperamental aspects may define clinically and genetically homogeneous bipolar disorder subtypes. Although that study differed from our present one in that they applied a bipolar patient sample as opposed to our general sample, none of the above SNPs or regions have been significant or suggestively significant in our present analysis.

In the present study, we analysed genome-wide associations for each of the five affective temperaments separately and given the significant moderate intercorrelations between them, all models were corrected for the effect of all other temperaments at SNP-based levels. In the next step, as assigning SNPs to genes purely based on their proximity yields only a limited insight into the complexity of the genetic background and little information on the whole spectrum of possible functional effects, and as the majority of identified SNPs are intronic or located in noncoding RNA introns and thus likely influence function of protein- or RNA-coding genes by mechanisms other than amino acid replacement, we carried out functional analysis using FUMA focusing on chromatin interactions and effects on gene expression in the brain. Here we discuss findings related to genes expressed in the brain or implicated in relevant psychiatric or psychological phenotypes in previous analyses.

\section{Genetic underpinnings of the anxious affective temperament}

The most robust finding of the study was identification of a novel intronic SNP in the ADGRB3 gene on chromosome 6, which survived correction for multiple testing in addition to 21 other intronic variants with suggestive $p$ values within this gene supporting its involvement in anxious temperament. The $A D G R B 3$ gene is expressed almost exclusively in the brain and encodes the Adhesion G protein-coupled receptor B3, or brain-specific angiogenesis inhibitor 3 (BAI3), a protein with a pivotal role in the central nervous system development, maintenance and plasticity, including regulating synaptic development, axon guidance, synapse formation, myelination, with high expression in the postsynaptic density, especially in the cerebellum, and crucial roles in Purkinje cell synaptogenesis, both during development and adulthood ${ }^{30-32}$. Specifically, the encoded protein controls synaptic connectivity of excitatory inputs, and several SNPs and copy number variations within this gene have previously been associated with different psychiatric disorders including bipolar disorder ${ }^{33}$, schizophrenia ${ }^{34}$ and addiction $^{35}$, as well as with traits such as impulsivity/negative urgency/ behavioural disinhibition ${ }^{36}$ and educational attainment ${ }^{37}$. $A D G R B 3$ shows high expression in the cerebellum and in hippocampal neurons where it acts as a regulator of synapse density and where its knockdown leads to significant impairment in dendrite morphogenesis in mouse models, whereas in a human study the clinical spectrum in patients with biallelic intragenic duplication in $A D G R B 3$ also included, among other symptoms, anxiety and mood instability, which are associated with hippocampal dysfunction $^{32,38,39}$ and which is specifically in line with our findings that variation in this gene shows association of anxious affective temperament. Further research, focusing on the manipulation of this gene in knockout rodent paradigms, as well as human studies focusing on its association with other anxiety-related endophenotypes and neuropsychiatric symptoms and illnesses should further validate the potential role of this gene and its variation in association with anxiety-related traits and disorders, and eventually the receptor encoded by this gene should be investigated as a potential treatment target.

In gene-based analyses for the anxious temperament, several genes reached suggestive significance after correction for multiple testing, some of which play a role in the central nervous system processes and thus may have potential relevance. Genes with a suggestive significance for the anxious temperament include $F Y N$, encoding the proto-oncogene tyrosine protein kinase fyn, which, among 
several other functions, plays a role in neuronal development and axon guidance, and is implicated in various neural functions and processes. Fyn kinase has, in previous studies, been implicated to play a role in the background of anger/aggression ${ }^{40}$ and in the pathophysiology of major depression $^{41,42}$, bipolar disorder ${ }^{43}$, schizophrenia ${ }^{44,45}$ and Parkinson's disease ${ }^{46,47}$, and has been suggested as a potential drug target in Alzheimer's disease ${ }^{48}$. PIP encoding prolactin-induced protein has also showed suggestive significance in association with anxious temperament. An SNP in the PIP gene in a previous GWAS has been found to be associated with unipolar depression (rs28672333) ${ }^{49}$. HECTD1, another gene suggestively associated with the anxious temperament in our present analysis, regulates the expression of $S N A I L^{50}$, which in turn regulates neural crest differentiation and neurogenesis ${ }^{51}$. Finally, ROGDI also emerged as a suggestive gene in association with anxious temperament. ROGDI encodes a typical leucine zipper protein with an unknown function, with highest expression in the adult brain ${ }^{52}$ and lower expression in the fetal brain and other tissues.

Furthermore, functional prediction analyses implicated top SNPs for the anxious temperament to be associated with the expression of various genes in the central nervous system in areas relevant both for emotional reactivity and affective illnesses, such as SLC35F2 in the DLPFC, hypothalamus and nucleus accumbens, and implicated in GWASs for unipolar depression, bipolar disorder, schizophrenia, attention deficit hyperactivity disorder (ADHD) and autism spectrum disorder ${ }^{53}$. Top SNPs for the anxious temperament also influence expression of $A C A T 1$ encoding the acetyl-CoA Acetyltransferase1, the major isoform in the brain, which has been implicated in several neurodegenerative disorders and has recently emerged as a promising target in the treatment of Alzheimer's disease ${ }^{54}$ and glioblastoma ${ }^{55}$. Top SNPs for the anxious temperament also regulate the expression of $E L M O D 1$, which has previously been associated with personality $^{56}$ and delayed discounting measures ${ }^{57}$.

\section{Genetic underpinnings of the cyclothymic affective temperament}

In SNP-based tests in case of the cyclothymic temperament, suggestive SNPs were located either on chromosome 2 within the ERBB4 gene or on chromosome 11, with 1 out of 6 of these latter SNPs in the NAP1L4 or SNORA genes. One more suggestive SNP was located in the CSMD1 gene.

$E R B B 4$ is a member of the Tyr kinase family and encodes a neuregulin surface receptor regulating development of the central nervous system including gene transcription, cell proliferation, differentiation, migration and apoptosis, expressed in interneurons in the frontal cortex and involved in postsynaptic modulation of GABAergic function ${ }^{58}$.
Variation in $E R B B 4$ has been reported in association with serotonin metabolite levels ${ }^{59}$, the openness personality trait of the five-factor model ${ }^{60}$, neuroticism ${ }^{61-63}$, positive affect/ wellbeing $^{63}$, intelligence ${ }^{64,65}$, educational attainment ${ }^{62}$, cognitive function ${ }^{37}$, loneliness/isolation/social interaction ${ }^{66-68}$ and depressed affect/unipolar depression/mood disorder ${ }^{61}$. Functional analysis indicated that top SNPs also take part in chromatin interactions with ERBB4 in the brain further underlying its association with cyclothymic temperament.

CSMD1, another gene with a suggestive SNP in the SNP-based tests for cyclothymic temperament, is expressed in the developing central nervous system and shows particular enrichment in the nerve growth cone, and has been suggested as a key regulator of complement activation and inflammation in the developing central nervous system $^{69}$. Variation in CSMD1 has been suggested in GWASs in association with several potentially relevant phenotypes including chronotype/morningness ${ }^{62,70-72}$, neuroticism $^{61}$, loneliness ${ }^{73}$, impulsivity/negative urgency/ behavioural disinhibition ${ }^{36}$, illegal drug consumption ${ }^{74}$, cannabis dependence ${ }^{75}$, alcohol consumption ${ }^{76}$, nicotine dependence $^{77,78}$, cognitive performance ${ }^{79}$, reaction time/ cognitive performance ${ }^{65}$, self-reported educational attainment $^{37}$, a combined analysis for five major psychiatric disorders including attention deficit/hyperactivity disorder (ADHD)/major depressive disorder/schizophrenia/autism spectrum disorder (ASD)/bipolar disorder ${ }^{53}$, aggressiveness in $\mathrm{ADHD}^{80}$, post-traumatic stress disorder (PTSD) ${ }^{81}$, schizophrenia/schizoaffective disorder/bipolar disorder ${ }^{82}$, psychosis-proneness $^{83}$, paliperidone-efficacy ${ }^{84}$, schizophrenia $^{44,45,85-92}$, suicide attempt in bipolar disorder ${ }^{93}$, eating disorder ${ }^{94}$ and in a common analysis of eight psychiatric disorders (anorexia nervosa, obsessive-compulsive disorder, ADHD, Tourette syndrome, unipolar depression, schizophrenia, autism spectrum disorder and bipolar disorder) $)^{94}$.

There were several suggestive hits for cyclothymic temperament at the gene level as well, including NAP1L4 where a functional effect was also observed and which has been mentioned at a suggestive significance level in association with major depression in a transcriptomewide analysis ${ }^{95}$. Top SNPs for cyclothymic temperament influenced brain expression of a few potentially relevant genes and participated in chromatin interactions, most notably with $E R B B 4$ and $S L C 36 A 4$, which latter encodes a sodium-independent electroneutral transporter for tryptophan, proline and alanine, and is also associated with chronotype $^{71}$.

\section{Genetic underpinnings of the depressive affective temperament}

At the SNP level, several suggestively significant SNPs for the depressive temperament were identified in the $S G C Z$ gene, which is expressed in several brain regions 
including highest expression in the cerebellum, basal ganglia and cortex, and has been implicated in the sarcoglycan complex and muscular dystrophy. $S G C Z$ has, in previous studies, been associated with some potentially relevant phenotypes including anxiety ${ }^{61,62}$, neuroticism $^{61,62,96}$, cognitive function ${ }^{65}$, mathematical ability ${ }^{37}$, intelligence ${ }^{64,97}$, self-reported educational attainment ${ }^{37}$, depressive symptom and response to antidepressant ${ }^{98}$, schizophrenia $^{99}$ and response to paliperidone ${ }^{84}$.

At the gene level, UGT2B7 encoding UDP-Glucuronosyltransferase-2B7, a phase II metabolic isoenzyme, also found in the brain showed a suggestive-level association with depressive temperament. For top SNPs in association with depressive temperament, we could not identify relevant effects on gene expression in the brain or chromatin interactions.

\section{Genetic underpinnings of the irritable temperament}

At the SNP level, suggestive SNPs for the irritable temperament were identified on chromosome 17 in the SHISA6 gene, which encodes a protein involved in the maintenance of high-frequency synaptic transmission in hippocampal CA3-CA1 synapses as an auxiliary $\alpha$-amino3-hydroxy-5-methyl-4-isoxazolepropionic acid (AMPA) receptor (AMPAR) subunit, and regulates postsynaptic AMPAR glutamate receptor immobilization keeping the channel in an activated state in the presence of glutamate, preventing synaptic depression and desensitization ${ }^{100}$. Variation in the SHISA6 gene in GWASs has been implicated in risk-taking behaviour/adventurousness ${ }^{101}$, insomnia $^{70}$, sleep duration ${ }^{102}$ and schizophrenia ${ }^{88}$. In gene-level analyses, suggestive hits included ENTPD6, which has been found to be associated with smoking status/smoking initiation ${ }^{103}$, and brain volume of the left pallidum ${ }^{104}$. In functional analyses, no effect of our top SNPs for irritable temperament emerged for influencing gene expression, but chromatin interactions were found with $N B E A$ and $R F C 3$ in the adult and fetal cortices. $N B E A$ encodes neurobeachin, lack of which protein in the $\mathrm{BEACH}$ domain in neurons was associated with a sharp reduction in synaptic responses as a consequence of reduced surface GABA-A and glutamate receptors, accumulation of immature AMPA receptors, and NMDA, kainite and GABA-A receptors not reaching the synapse, suggesting that Nbea plays a role in regulating basal neurotransmission and targeting of neurotransmitter receptors to synapses ${ }^{105}$. NBEA variations in GWASs have been reported in antipsychotic response in schizophrenia $^{106}$, nicotine dependence ${ }^{78}$, self-reported educational attainment ${ }^{37}, \mathrm{PTSD}^{76}$, and cognitive function ${ }^{37}$.

\section{Genetic underpinnings of the hyperthymic temperament}

At the SNP level, suggestive hits for the hyperthymic temperament were identified on chromosome 4,8 and 20 , and on chromosome 6 in the region of CDSN and PSORSC1 genes. PSORSC1 is expressed in the brain and has been associated in previous GWASs with neuroti$\operatorname{cism}^{63}$, risk-taking behaviour ${ }^{101}$, wellbeing/positive affect and wellbeing/life satisfaction, and subjective wellbeing ${ }^{63,107}$, depressive symptom measurement ${ }^{63}$, schizophrenia $^{44,88,108}$, a broad depression or schizophrenia phenotype ${ }^{109}$ and autism spectrum disorder ${ }^{108}$. On the gene level, suggestively significant hits for hyperthymic temperament included SNX6 and CANT1 with high expression in the brain, but no relevant phenotypic associations in previous studies. Top SNPs associated with hyperthymic temperament participated in chromatin interactions with a few brain-expressed genes but without previous association with relevant phenotypes.

\section{Potential transdiagnostic and pathoplastic nature of affective temperaments}

Our results indicate that the five distinct affective temperaments have non-overlapping genetic backgrounds; however, several suggestively significant hits were observable for genes which have previously been implicated in association with relevant psychological traits related to the development of mood disorders such as neuroticism, impulsiveness, aggression or wellbeing, as well as in other psychiatric disorders. These two findings, namely distinct genetic underpinnings of the five distinct temperaments and the same disorders these distinct genes confer a risk for, suggest a potentially transdiagnostic endophenotypic nature of the TEMPS affective temperaments. If considering psychiatric disorders other than mood disorders, schizophrenia has been found to have common genetic risk factors with all five temperaments. Addiction may share some genetics with anxious, cyclothymic and irritable temperaments. Autism spectrum disorder has been implicated in relation to risk genes for anxious and hyperthymic temperaments, and PTSD in genes for cyclothymic and irritable temperaments. Distinct temperaments with their distinct genetic underpinnings may represent distinct subgroups within these aetiologically heterogeneous disorders. Stratification of patients or at-risk individuals according to these welldefined endophenotypic pathways, from certain genes through certain affective temperaments to the equifinal endpoint of the disorder, may help in the personalization of preventive and therapeutic strategies for these disorders. However, further longitudinal studies are needed to clarify the mediatory role of affective temperaments between genes and the emergence of these various disorders.

A major weakness of our study is the small sample size highlighted by terming the present analysis a pilot study and which is in part balanced by the careful sample characterization and precise attainment of the investigated 
phenotype, and multiple analytical approaches focusing on functional analyses besides SNP and gene levels. Furthermore, although precise deep phenotyping of affective temperaments is a strength of the present analysis, affective temperaments are measured based on self-report and are ascertained cross-sectionally, and thus may be influenced by state-like factors such as mood or possible mood disorder episode.

In conclusion, our GWAS focusing on affective temperaments, besides several suggestively significant hits at SNP and gene levels, have most notably identified a new genome-wide significant variant in association with anxious temperament in AGDRB3 gene. Furthermore, although the five affective temperament phenotypes have non-overlapping genetic backgrounds, we identified several suggestively significant variants previously associated with psychological traits implicated in the development of both mood and other types of psychiatric disorders, implicating the potential transdiagnostic endophenotypic nature of affective temperaments. In the future, our findings may help pave the way for personalized and precision approaches for both prevention and intervention strategies across various psychiatric disorders. Our results should further be tested in larger samples in genome-wide analytical approaches for related phenotypes and candidate gene studies in well-characterized and phenotyped samples, to more precisely establish the role of our identified genes and variants in mood disorders or high-risk states for the development of affective illness, as well as its role as a potential target of therapeutic interventions. Longitudinal studies should also test our findings to understand the potential mediative role of affective temperaments between genes and emergence of various psychiatric disorders.

\footnotetext{
Acknowledgements

The study was supported by the Sixth Framework Program of the European Union (NewMood, LSHM-CT-2004-503474); by the Hungarian Brain Research Program (Grants KTIA_13_NAP-A-II/14 and 2017-1.2.1-NKP-2017-00002), and the National Development Agency (Grant KTIA_NAP_13-1-2013-0001); by the Hungarian Academy of Sciences, Hungarian National Development Agency, Semmelweis University and the Hungarian Brain Research Program (Grant KTIA_NAP_13-2-2015-0001, MTA-SE-NAP B Genetic Brain Imaging Migraine Research Group); by the Hungarian Academy of Sciences (MTA-SE Neuropsychopharmacology and Neurochemistry Research Group); by OTKA 119866; by the NRDI Fund (TKP2020 IES, Grant Number BME-IE-BIO) based on the charter of bolster issued by the NRDI Office under the auspices of the Ministry for Innovation and Technology; by TAMOP-4.2.1.B-09/1/KMR-20100001; by the New National Excellence Program of The Ministry for Innovation and Technology from the source of the National Research, Development and Innovation Fund (ÚNKP-20-4-II-SE-9; ÚNKP-20-3-II-SE-51); by the National Research, Development and Innovation Office, Hungary (2019-2.1.7-ERA-NET2020-00005), under the frame of ERA PerMed (ERAPERMED2019-108); by the Ministry of Innovation and the National Research, Development and Innovation Office within the framework of the Artificial Intelligence National Laboratory Programme; and by the Thematic Excellence Programme (Tématerületi Kiválósági Program, 2020-4.1.1.-TKP2020) of the Ministry for Innovation and Technology in Hungary, within the framework of the Neurology and Translational Biotechnology thematic programmes of the Semmelweis University. P. P. is an international research fellow of Japan Society
}

for the Promotion of Science (Postdoctoral Fellowships for Research in Japan, standard program, P20809). X.G. is a recipient of the Janos Bolyai Research Fellowship of the Hungarian Academy of Sciences.

\section{Author details}

'NAP-2-SE New Antidepressant Target Research Group, Hungarian Brain Research Program, Semmelweis University, Budapest, Hungary. ${ }^{2}$ Department of Psychiatry and Psychotherapy, Faculty of Medicine, Semmelweis University, Budapest, Hungary. ${ }^{3}$ Department of Pharmacodynamics, Faculty of Pharmacy, Semmelweis University, Budapest, Hungary. ${ }^{4}$ Department of Forensic and Insurance Medicine, Faculty of Medicine, Semmelweis University, Budapest, Hungary. ${ }^{5}$ Department of Measurement and Information Systems, Budapest University of Technology and Economics, Budapest, Hungary. ${ }^{6}$ Abiomics Europe Ltd, Budapest, Hungary. ${ }^{7}$ SE-NAP 2 Genetic Brain Imaging Migraine Research Group, Hungarian Brain Research Program, Semmelweis University, Budapest, Hungary. ${ }^{8}$ Bioinformatics Center, Institute for Chemical Research, Kyoto University, Uji, Japan. ${ }^{9}$ Social, Genetic and Developmental Psychiatry Centre, King's College London, London, UK

\section{Data availability}

Datasets presented in this study are available at https://doi.org/10.6084/m9. figshare.13498536.v1.

\section{Conflict of interest}

G.B. has received grant funding from and served as a consultant to Eli Lilly, has received honoraria from Illumina and has served on advisory boards for Otsuka. All other authors have indicated they have no conflicts of interest to disclose.

\section{Publisher's note}

Springer Nature remains neutral with regard to jurisdictional claims in published maps and institutional affiliations.

Supplementary information The online version contains supplementary material available at https://doi.org/10.1038/s41398-021-01436-1.

Received: 29 December 2020 Revised: 29 April 2021 Accepted: 5 May 2021 Published online: 01 June 2021

\section{References}

1. Nakka, P., Raphael, B. J. \& Ramachandran, S. Gene and network analysis of common variants reveals novel associations in multiple complex diseases. Genetics 204, 783-798 (2016).

2. Friedrich, M. J. Depression is the leading cause of disability around the world. JAMA 317, 1517 (2017).

3. Border, R. et al. No support for historical candidate gene or candidate geneby-interaction hypotheses for major depression across multiple large samples. Am. J. Psychiat 176, 376-387 (2019).

4. Howard, D. M. et al. Genome-wide meta-analysis of depression identifies 102 independent variants and highlights the importance of the prefrontal brain regions. Nat. Neurosci. 22, 343-352 (2019).

5. Ostergaard, S. D., Jensen, S. O. W. \& Bech, P. The heterogeneity of the depressive syndrome: when numbers get serious. Acta Psychiatr. Scand. 124, 495-496 (2011).

6. Gonda, X. et al. Genetic variants in major depressive disorder: from pathophysiology to therapy. Pharmacol. Ther. https://doi.org/10.1016/j. pharmthera.2018.09.002 (2018).

7. Han, K. M., De Berardis, D., Fornaro, M. \& Kim, Y. K. Differentiating between bipolar and unipolar depression in functional and structural MRI studies. Prog. Neuropsychopharmacol. Biol. Psychiatry 91, 20-27 (2019).

8. De Berardis, D. et al. Alexithymia, resilience, somatic sensations and their relationships with suicide ideation in drug naive patients with first-episode major depression: An exploratory study in the "real world" everyday clinical practice. Early Inter. Psychiatry 14, 336-342 (2020).

9. Gold, P. W. \& Chrousos, G. P. Melancholic and atypical subtypes of depression represent distinct pathophysiological entities: $\mathrm{CRH}$, neural circuits, and the diathesis for anxiety and depression. Mol. Psychiatry 18, 632-634 (2013). 
10. Orsolini, L. et al. Understanding the complex of suicide in depression: from research to clinics. Psychiatry Investig. 17, 207-221 (2020).

11. Flint, J. \& Munafo, M. R. The endophenotype concept in psychiatric genetics. Psychol. Med. 37, 163-180 (2007).

12. Akiskal, H. S. \& Akiskal, K. K. In search of Aristotle: temperament, human nature, melancholia, creativity and eminence. J. Affect. Disord. 100, 1-6 (2007)

13. Rihmer, Z., Akiskal, K. K., Rihmer, A. \& Akiskal, H. S. Current research on affective temperaments. Curr. Opin. Psychiatr. 23, 12-18 (2010).

14. Greenwood, T. A., Akiskal, H. S., Akiskal, K. K., Study, B. G. \& Kelsoe, J. R. Genome-wide association study of temperament in bipolar disorder reveals significant associations with three novel loci. Biol. Psychiatry 72, 303-310 (2012).

15. Deakin, B. New molecules for mood disorders; the NewMood EU project. Eur. Neuropsychopharmacol. 19, s107-s108 (2009).

16. Deakin, J. F., Harro, J. \& Anderson, I. M. NewMood: a productive European model of collaboration for translational research in depression. Eur. Neuropsychopharmacol. 21, 1-2 (2011).

17. Deakin, J. F. et al. From animals to man: overview and main findings from the NewMood project. Eur. Neuropsychopharmacol. 19, S214 (2009).

18. Rozsa, S. et al. A study of affective temperaments in Hungary: Internal consistency and concurrent validity of the TEMPS-A against the TO and NEO-PIR. J. Affect. Disord. 106, 45-53 (2008).

19. Freeman, B. et al. DNA from buccal swabs recruited by mail: evaluation of storage effects on long-term stability and suitability for multiplex polymerase chain reaction genotyping. Behav. Genet. 33, 67-72 (2003).

20. Coleman, J. R. et al. Quality control, imputation and analysis of genome-wide genotyping data from the Illumina HumanCoreExome microarray. Brief. Funct. Genomics 15, 298-304 (2016).

21. Eszlari, N. et al. Genome-wide association analysis reveals KCTD12 and miR383-binding genes in the background of rumination. Transl. Psychiatry 9, 119 (2019).

22. Watanabe, K., Taskesen, E., van Bochoven, A. \& Posthuma, D. Functional mapping and annotation of genetic associations with FUMA. Nat. Commun. 8, 1826 (2017).

23. Gibson, J. et al. A meta-analysis of genome-wide association studies of epigenetic age acceleration. PLoS Genet. 15, e1008104 (2019).

24. GTEx Consortium. Human genomics. The Genotype-Tissue Expression (GTEx) pilot analysis: multitissue gene regulation in humans. Science $\mathbf{3 4 8}, 648-660$ (2015).

25. GTEx Consortium. Genetic effects on gene expression across human tissues. Nature 550, 204-213 (2017).

26. Ramasamy, A. et al. Genetic variability in the regulation of gene expression in ten regions of the human brain. Nat. Neurosci. 17, 1418-1428 (2014).

27. $\mathrm{Ng}, \mathrm{B}$. et al. An $\mathrm{xQTL}$ map integrates the genetic architecture of the human brain's transcriptome and epigenome. Nat. Neurosci. 20, 1418-1426 (2017).

28. Fromer, M. et al. Gene expression elucidates functional impact of polygenic risk for schizophrenia. Nat. Neurosci. 19, 1442-1453 (2016).

29. Giusti-Rodríguez, P. et al. Using three-dimensional regulatory chromatin interactions from adult and fetal cortex to interpret genetic results for psychiatric disorders and cognitive traits. Preprint at bioRxiv https:/doi.org/ 10.1101/406330 (2019).

30. Sigoillot, S. M. et al. The secreted protein C1QL1 and its receptor BAI3 contro the synaptic connectivity of excitatory inputs converging on cerebellar Purkinje cells. Cell Rep. 10, 820-832 (2015).

31. Kakegawa, W. et al. Anterograde Clql1 signaling is required in order to determine and maintain a single-winner climbing fiber in the mouse cerebellum. Neuron 85, 316-329 (2015).

32. Scuderi, $C$. et al. Biallelic intragenic duplication in ADGRB3 (BAl3) gene associated with intellectual disability, cerebellar atrophy, and behavioral disorder. Eur. J. Hum. Genet. 27, 594-602 (2019).

33. McCarthy, M. J., Nievergelt, C. M., Kelsoe, J. R. \& Welsh, D. K. A survey of genomic studies supports association of circadian clock genes with bipolar disorder spectrum illnesses and lithium response. PLOS ONE 7, e32091 (2012).

34. DeRosse, P. et al. The genetics of symptom-based phenotypes: toward a molecular classification of schizophrenia. Schizophr. Bull. 34, 1047-1053 (2008).

35. Liu, Q. R. et al. Addiction molecular genetics: 639,401 SNP whole genome association identifies many "cell adhesion" genes. Am. J. Med. Genet. B Neuropsychiatr. Genet. 141B, 918-925 (2006).

36. Sanchez-Roige, S. et al. Genome-wide association studies of impulsive personality traits (BIS-11 and UPPSP) and drug experimentation in up to 22,861 adult research participants identify loci in the CACNA1I and CADM2 genes. J. Neurosci. 39, 2562-2572 (2019).

37. Lee, J. J. et al. Gene discovery and polygenic prediction from a genome-wide association study of educational attainment in 1.1 million individuals. Nat Genet. 50, 1112-1121 (2018).

38. Rubin, R. D., Watson, P. D., Duff, M. C. \& Cohen, N. J. The role of the hippocampus in flexible cognition and social behavior. Front. Hum. Neurosci. 8 , 742 (2014).

39. Lanoue, $V$. et al. The adhesion-GPCR BAl3, a gene linked to psychiatric disorders, regulates dendrite morphogenesis in neurons. Mol. Psychiatry $\mathbf{1 8}$ 943-950 (2013).

40. Mick, E. et al. Genome-wide association study of proneness to anger. PLoS ONE 9, e87257 (2014).

41. Kulikova, E. \& Kulikov, A. Striatal-enriched tyrosine protein phosphatase (STEP) in the mechanisms of depressive disorders. Curr. Protein Pept. Sci. 18, 1152-1162 (2017).

42. Mao, L. M. \& Wang, J. Q. Tyrosine phosphorylation of glutamate receptors by non-receptor tyrosine kinases: roles in depression-like behavior. Neurotransmitter 3, e1118 (2016).

43. Stahl, E. A. et al. Genome-wide association study identifies 30 loci associated with bipolar disorder. Nat. Genet. 51, 793-803 (2019).

44. Ikeda, M. et al. Genome-wide association study detected novel susceptibility genes for schizophrenia and shared trans-populations/diseases genetic effect. Schizophr. Bull. 45, 824-834 (2019).

45. Li, Z. et al. Genome-wide association analysis identifies 30 new susceptibility loci for schizophrenia. Nat. Genet. 49, 1576-1583 (2017).

46. Panicker, N., Kanthasamy, A. \& Kanthasamy, A. G. Fyn amplifies NLRP3 inflammasome signaling in Parkinson's disease. Aging 11, 5871-5873 (2019).

47. Foo, J. N. et al. Identification of risk loci for Parkinson disease in Asians and comparison of risk between Asians and Europeans: a genome-wide association study. JAMA Neurol. 77, 746-754 (2020).

48. Nygaard, H. B. Targeting Fyn kinase in Alzheimer's disease. Biol. Psychiatry 83, 369-376 (2018).

49. Coleman, J. R. I. et al. Genome-wide gene-environment analyses of major depressive disorder and reported lifetime traumatic experiences in UK Biobank. Mol. Psychiatry 25, 1430-1446 (2020).

50. Wang, X., De Geyter, C., Jia, Z., Peng, Y. \& Zhang, H. HECTD1 regulates the expression of SNAIL: implications for epithelialmesenchymal transition. Int. J. Oncol. 56, 1186-1198 (2020).

51. York, J. R., Zehnder, K., Yuan, T., Lakiza, O. \& McCauley, D. W. Evolution of Snailmediated regulation of neural crest and placodes from an ancient role in bilaterian neurogenesis. Dev. Biol. 453, 180-190 (2019).

52. Mory, A. et al. A nonsense mutation in the human homolog of Drosophila rogdi causes Kohlschutter-Tonz syndrome. Am. J. Hum. Genet. 90, 708-714 (2012).

53. Cross-Disorder Group of the Psychiatric Genomics Consortium Identification of risk loci with shared effects on five major psychiatric disorders: a genomewide analysis. Lancet 381, 1371-1379 (2013).

54. Alavez-Rubio, J. S. \& Juarez-Cedillo, T. ACAT1 as a therapeutic target and its genetic relationship with Alzheimer's disease. Curr. Alzheimer Res. 16, 699-709 (2019).

55. Shibuya, K. et al. Brain targeting of acyl-CoA:cholesterol O-acyltransferase-1 inhibitor K-604 via the intranasal route using a hydroxycarboxylic acid solution. ACS Omega 4, 16943-06955 (2019).

56. Eriksson, N. et al. Web-based, participant-driven studies yield novel genetic associations for common traits. PLoS Genet. 6, el000993 (2010).

57. Mackillop, J. et al. Genetic influences on delayed reward discounting: a genome-wide prioritized subset approach. Exp. Clin. Psychopharmacol. 27, 29-37 (2019).

58. Neddens, J. et al. Conserved interneuron-specific ErbB4 expression in frontal cortex of rodents, monkeys, and humans: implications for schizophrenia. Biol. Psychiatry 70, 636-645 (2011).

59. Rhee, E. P. et al. A genome-wide association study of the human metabolome in a community-based cohort. Cell Metab. 18, 130-143 (2013).

60. Kim, H. N. et al. Genome-wide association study of the five-factor model of personality in young Korean women. J. Hum. Genet. 58, 667-674 (2013).

61. Nagel, M. et al. Meta-analysis of genome-wide association studies for neuroticism in 449,484 individuals identifies novel genetic loci and pathways. Nat. Genet. 50, 920-927 (2018).

62. Kichaev, G. et al. Leveraging polygenic functional enrichment to improve GWAS power. Am. J. Hum. Genet. 104, 65-75 (2019). 
63. Baselmans, B. M. L. et al. Multivariate genome-wide analyses of the wellbeing spectrum. Nat. Genet. 51, 445-451 (2019).

64. Hill, W. D. et al. A combined analysis of genetically correlated traits identifies 187 loci and a role for neurogenesis and myelination in intelligence. Mol. Psychiatry 24, 169-181 (2019).

65. Davies, G. et al. Study of 300,486 individuals identifies 148 independent genetic loci influencing general cognitive function. Nat. Commun. 9, 2098 (2018).

66. Nagel, M., Watanabe, K., Stringer, S., Posthuma, D. \& van der Sluis, S. Item-level analyses reveal genetic heterogeneity in neuroticism. Nat. Commun. 9, 905 (2018).

67. Day, F. R., Ong, K. K. \& Perry, J. R. B. Elucidating the genetic basis of social interaction and isolation. Nat. Commun. 9, 2457 (2018).

68. Abdellaoui, A. et al. Phenome-wide investigation of health outcomes associated with genetic predisposition to loneliness. Hum. Mol. Genet. 28 3853-3865 (2019).

69. Kraus, D. M. et al. CSMD1 is a novel multiple domain complement-regulatory protein highly expressed in the central nervous system and epithelial tissues. J. Immunol. 176, 4419-4430 (2006).

70. Jansen, P. R. et al. Genome-wide analysis of insomnia in 1,331,010 individuals identifies new risk loci and functional pathways. Nat. Genet. 51, 394-403 (2019).

71. Jones, S. E. et al. Genome-wide association analyses of chronotype in 697,828 individuals provides insights into circadian rhythms. Nat. Commun. 10 https://doi.org/10.1038/s41467-018-08259-7 (2019).

72. $\mathrm{Hu}, \mathrm{Y}$. et al. WAS of 89,283 individuals identifies genetic variants associated with self-reporting of being a morning person. Nat. Commun. 7, 10448 (2016).

73. Gao, J. et al. Genome-wide association study of loneliness demonstrates a role for common variation. Neuropsychopharmacology 42, 811-821 (2017).

74. McGue, M. et al. A genome-wide association study of behavioral disinhibition. Behav. Genet. 43, 363-373 (2013).

75. Sherva, R. et al. Genome-wide association study of cannabis dependence severity, novel risk variants, and shared genetic risks. JAMA Psychiatry 73, 472-480 (2016).

76. Gelernter, J. et al. Genome-wide association study of post-traumatic stress disorder reexperiencing symptoms in $>165,000$ US veterans. Nat. Neurosci. 22, 1394-1401 (2019).

77. Hällfors, J. et al. Genome-wide association study in Finnish twins highlights the connection between nicotine addiction and neurotrophin signaling pathway. Addict. Biol. 24, 549 (2018)

78. Gelernter, J. et al. Genome-wide association study of nicotine dependence in American populations: identification of novel risk loci in both AfricanAmericans and European-Americans. Biol. Psychiatry 77, 493-503 (2015).

79. Cirulli, E. T. et al. Common genetic variation and performance on standardized cognitive tests. Eur. J. Hum. Genet. 18, 815-820 (2010).

80. Brevik, E. J. et al. Genome-wide analyses of aggressiveness in attentiondeficit hyperactivity disorder. Am. J. Med. Genet. B Neuropsychiatr. Genet. 171, 733-747 (2016).

81. Nievergelt, C. M. et al. Genomic predictors of combat stress vulnerability and resilience in U.S. Marines: a genome-wide association study across multiple ancestries implicates PRTFDC1 as a potential PTSD gene. Psychoneuroendocrinology 51, 459-471 (2015)

82. Sleiman, P. et al. GWAS meta analysis identifies TSNARE1 as a novel schizophrenia/bipolar susceptibility locus. Sci. Rep. 3, 3075 (2013).

83. Ortega-Alonso, A. et al. Genome-wide association study of psychosis proneness in the Finnish population. Schizophr. Bull. 43, 1304-1314 (2017).

84. Li, Q. et al. Genome-wide association study of paliperidone efficacy. Pharmacogenet. Genomics 27, 7-18 (2017).

85. Bergen, S. E. et al. Genome-wide association study in a Swedish population yields support for greater CNV and MHC involvement in schizophrenia compared with bipolar disorder. Mol. Psychiatry 17, 880-886 (2012).

86. Lam, M. et al. Comparative genetic architectures of schizophrenia in East Asian and European populations. Nat. Genet. 51, 1670-1678 (2019).

87. $\mathrm{Yu}, \mathrm{H}$. et al. Common variants on 2p16.1, 6p22.1 and 10q24.32 are associated with schizophrenia in Han Chinese population. Mol. Psychiatry 22, 954-960 (2017).
88. Goes, F. S. et al. Genome-wide association study of schizophrenia in Ashkenazi Jews. Am. J. Med. Genet. B Neuropsychiatr. Genet. 168, 649-659 (2015).

89. Schizophrenia Working Group of the Psychiatric Genomics Consortium Biological insights from 108 schizophrenia-associated genetic loci. Nature 511, 421-427 (2014).

90. Schizophrenia Psychiatric Genome-Wide Association Study Consortium Genome-wide association study identifies five new schizophrenia loci. Nat. Genet 43, 969-976 (2011).

91. Periyasamy, S. et al. Association of schizophrenia risk with disordered niacin metabolism in an Indian genome-wide association study. JAMA Psychiatry https://doi.org/10.1001/jamapsychiatry.2019.1335 (2019).

92. Pardinas, A. F. et al. Common schizophrenia alleles are enriched in mutationintolerant genes and in regions under strong background selection. Nat. Genet. 50, 381-389 (2018).

93. Mullins, N. et al. GWAS of suicide attempt in psychiatric disorders and association with major depression polygenic risk scores. Am. J. Psychiatry 176, 651-660 (2019).

94. Cross Disorder Group of the Psychiatric Genomics Consortium Genomic relationships, novel loci, and pleiotropic mechanisms across eight psychiatric disorders. Cell 179, 1469-1482 (2019).

95. Dall'Aglio, L., Lewis, C. M. \& Pain, O. Delineating the genetic component of gene expression in major depression. Biol. Psychiatry 89, 627-636 (2021).

96. Luciano, M. et al. Association analysis in over 329,000 individuals identifies 116 independent variants influencing neuroticism. Nat. Genet. 50, 6-11 (2018).

97. Savage, J. E. et al. Genome-wide association meta-analysis in 269,867 individuals identifies new genetic and functional links to intelligence. Nat. Genet. 50, 912-919 (2018).

98. Fabbri, $\mathrm{C}$. et al. Genome-wide association study of treatment-resistance in depression and meta-analysis of three independent samples. Br. J. Psychiatry 214, 36-41 (2019).

99. Ikeda, M. et al. Evidence for shared genetic risk between methamphetamineinduced psychosis and schizophrenia. Neuropsychopharmacology $\mathbf{3 8}$ 1864-1870 (2013).

100. Klaassen, R. V. et al. Shisa6 traps AMPA receptors at postsynaptic sites and prevents their desensitization during synaptic activity. Nat. Commun. 7, https://doi.org/10.1038/ncomms10682. www.nature.com/ naturecommunications (2016).

101. Karlsson Linner, R. et al. Genome-wide association analyses of risk tolerance and risky behaviors in over 1 million individuals identify hundreds of loci and shared genetic influences. Nat. Genet. 51, 245-257 (2019).

102. Dashti, H. S. et al. Genome-wide association study identifies genetic loci for self-reported habitual sleep duration supported by accelerometer-derived estimates. Nat. Commun. 10, 1100 (2019).

103. Liu, M. et al. Association studies of up to 1.2 million individuals yield new insights into the genetic etiology of tobacco and alcohol use. Nat. Genet. 51, 237-244 (2019).

104. Zhao, B. et al. Genome-wide association analysis of 19,629 individuals identifies variants influencing regional brain volumes and refines their genetic co-architecture with cognitive and mental health traits. Nat. Genet. 51, 1637-1644 (2019).

105. Nair, R. et al. Neurobeachin regulates neurotransmitter receptor trafficking to synapses. J. Cell Biol. 200, 61-80 (2013).

106. $\mathrm{Yu}, \mathrm{H}$. et al. Five novel loci associated with antipsychotic treatment response in patients with schizophrenia: a genome-wide association study. Lancet Psychiatry 5, 327-338 (2018).

107. Turley, P. et al. Multi-trait analysis of genome-wide association summary statistics using MTAG. Nat. Genet. 50, 229-237 (2018).

108. Autism Spectrum Disorders Working Group of The Psychiatric Genomics Consortium Meta-analysis of GWAS of over 16,000 individuals with autism spectrum disorder highlights a novel locus at 10q24.32 and a significant overlap with schizophrenia. Mol. Autism 8, 21 (2017).

109. Amare, A. T. et al. Bivariate genome-wide association analyses of the broad depression phenotype combined with major depressive disorder, bipolar disorder or schizophrenia reveal eight novel genetic loci for depression. Mol. Psychiatry 25, 1420-1429 (2020). 